\title{
Inference on the dynamics of the COVID pandemic from observational data
}

\author{
Satarupa Bhattacharjee ${ }^{\mathrm{a}}$, Shuting Liao ${ }^{\mathrm{b}}$, \\ Debashis Paul ${ }^{\mathrm{a}}$, Sanjay Chaudhuri $\mathrm{c}^{*}$ \\ a Department of Statistics, University of California, Davis \\ ${ }^{b}$ Graduate Group in BioStatistics, University of California, Davis \\ ${ }^{c}$ Department of Statistics and Applied Probability, National University of Singapore
}

\begin{abstract}
We describe a time dependent stochastic dynamic model in discrete time for the evolution of the COVID-19 pandemic in various states of USA. The proposed multicompartment model is expressed through a system of difference equations that describe their temporal dynamics. Various compartments in our model is connected to the social distancing measures and diagnostic testing rates. A nonparametric estimation strategy is employed for obtaining estimates of interpretable temporally static and dynamic epidemiological rate parameters. The confidence bands of the parameters are obtained using a residual bootstrap procedure. A key feature of the methodology is its ability to estimate latent compartments such as the trajectory of the number of asymptomatic but infected individuals which are the key vectors of COVID-19 spread. The nature of the disease dynamics is further quantified by the proposed epidemiological markers, which use estimates of such key latent compartments.
\end{abstract}

KEY WORDS: COVID-19; Stochastic Dynamic Model; Nonparametric Inference; Multiple Compartments.

E-mail address: * Corresponding author: stasc@nus.edu.sg. 
medRxiv preprint doi: https://doi.org/10.1101/2021.02.01.21250936; this version posted February 3, 2021. The copyright holder for this preprint

The novel coronavirus has been ravaging the world since early 2020. First identified in Wuhan, Hubei Province, China, the epidemic has since spread to every corner of the world. As of January 25, 2021 [1], more than 99 million people have been infected, out of which more than 2.1 million have died of the disease. The World Health Organization declared the situation a pandemic on March 11, 2020. Since then various parts of the world have gone through multiple waves surges in the number of new infections. The pandemic has severely affected the world economy. Repeated lockdowns, travel restrictions, and other measures of containment have severely impacted the economy of many countries, stretched healthcare systems to the extreme, and caused mental health crisis for large chunks of the populations.

The new pathogen (SARS-CoV-2) which causes the disease [2] is mostly unknown in terms of its infectivity and clinical profile. It is well-known that the infection primarily spreads through infected but asymptomatic people. The number of such people remains unknown. The reported number is based on symptomatic or positively tested persons, which grossly underestimates of the true value. Because of the undetermined denominator effect, important epidemiological markers like the death rate, hospitalisation rate etc remains non-determinable from the observed data. Various estimates [3, 4, 5, 6, 7] of these markers have been postulated by many authors. Mathematical modelling and quantification of the epidemiological parameters [8, 9, 10, 11, 12, 13] of the pandemic have been crucial in understanding and interpreting the transmission dynamics from the perspective of public health researchers and policymakers around the globe [14, 15, 16, 17].

A number of popular compartmental epidemiological models e.g. SIR, SEIR, SIRD etc. have been employed to describe the dynamics of COVID-19 [18, 19, 20, 21]. Such models yield estimates of epidemilogical markers such as the basic reproduction number $\left(R_{0}\right)$, and 
medRxiv preprint doi: https://doi.org/10.1101/2021.02.01.21250936; this version posted February 3, 2021. The copyright holder for this preprint

Inference on COVID-19 dynamics
various doubling and case fatality rates that are indicators of the disease growth pattern [22, 23]. Prediction of epidemiological characteristics and transmission patterns in this context have also attracted major attention [24, 25, 26, 27]. Advanced statistical methods have been employed in forecasting the number of cases worldwide [28] or quantifying the effects of prevention mechanisms like social distancing [29, 30, 31, 32, 33, 34], public gathering, and travel restrictions [35, 36, 37] for various countries. Due to the difference in analytical methods and assumptions, the parameter estimates describing COVID-19 dynamics vary widely. This variability is also reflected in the estimates of the effectiveness of public health interventions implemented worldwide. Most epidemiological models of disease transmission are simplistic and use time-invariant transmission rates. However, in reality, due to mitigation efforts and the evolving nature of the infection mechanism, such rates become temporally dynamic. Furthermore, most SIER-type models exclude the effects of testing and subsequent quarantining, and occasionally, even hospitalization. Such practices fail to adequately account for the size of the susceptible population and therefore tend to provide unreliable estimates of the number of asymptomatic persons infected by COVID-19 in the population.

We propose a detailed discrete time semiparametric stochastic dynamic model for COVID-19 spread. The model is expressed through a system of difference equations connecting various interpretable compartments in the disease dynamics such as individuals who are susceptible, asymptomatic but infected, quarantined, hospitalized, dead and have recovered from the disease. We introduce interpretable time-varying parameters to reflect various temporally dynamic rates. Our model also includes available information on the number of tests. On the other hand, the proposed model does not make restrictive and often untestable distributional assumptions about compartments or parameters that are commonplace in various probablistic models for the epidemiological dynamics. 
We employ nonlinear nonparametric regression techniques through a profiling based estimation procedure to estimate the model parameters and the number of people in different compartments. Using residual bootstrap based techniques, we also provide point-wise confidence intervals (bands) for the time-invariant (time-varying) parameters. The proposed model and estimation procedure relies on linear kernel weighting and fairly low dimensional optimization, thus avoiding Markov Chain Monte Carlo and other computationally expensive methods employed by Bayesian inference schemes for standard epidemiological models. Therefore, the estimates can be obtained almost instantaneously. Another key feature of our method is the ability of identifying and estimating unobservable quantities such as the actual number of asymptomatic but infected people at any given time. The estimated trajectory of the infected but asymptomatic population over time, its doubling rate, the true case fatality rate, and an analogue of the basic reproduction rate are crucial in interpreting the time-dynamics of the pandemic. They have important implications for policy decisions regarding appropriate mitigation strategies.

\section{A Multi-compartment Model for Disease Spread}

We consider a closed population without emmigration or immigration and propose a model for the Covid-19 pandemic spread in terms of various observable and partially or totally unobservable compartments.

Suppose at time $t, C_{t}, D_{t}, T_{t}$, respectively, denote the number of confirmed cases, number of deaths due to the disease and the number of tests performed up to time $t$. These variables are non-decreasing cumulative counts and are generally fully observed. The number of hospitalised persons due to Covid-19 infection at time $t$ (denoted $H_{t}$ ) is also generally observed (see Section 3.2 for more detail). Furthermore, we observe $Q_{t}$, the 
number of asymptomatic individuals who are in quarantine at time $t$. These individuals have been tested positive, but show no significant symptoms requiring hospitalisation.

The most crucial unobserved compartment is $A_{t}$, i.e. the number of infected but asymptomatic individuals at time $t$. It is well known that the people in this group are primary spreaders of the disease. Furthermore, due to under-reporting, the number of confirmed cases would be a fraction of $A_{t}$. Since we do not observe how many in the population are currently infected, the number of susceptible individuals at time $t$, (denoted $S_{t}$ ) is also unobserved.

The number of recovered individuals (denoted $R_{t}$ ) up to time $t$ can be partially observed. To understand this, note that the recoveries from quarantine centres and hospitals, (denoted $R_{t}^{Q}$ and $R_{t}^{H}$ respectively) are reported, though not necessarily separately (see Supplement Section S2., for the case when $R_{t}^{Q}$ and $R_{t}^{H}$ are reported separately). But since $A_{t}$ is unobserved, the number of asymptomatic but infected people who recover without being quarantined or hospitalised (denoted $R_{t}^{A}$ ) cannot be observed. That is, even though $R_{t}^{\text {reported }}=R_{t}^{Q}+R_{t}^{H}$ is available from the data, the total recovery $R_{t}$ is not.

The proposed disease propagation model are based of the following assumptions:

A1 Only an asymptomatic individual who is not either in quarantine or in hospital can transmit the disease to a susceptible individual.

A2 People who recover from the disease are immune from subsequent infection.

A3 False positive rate for the test is negligible, so that if somebody is confirmed to be positive, then he/she is assumed to be infected.

A4 Anybody who shows significant symptoms, whether being in quarantine or not, is immediately hospitalized, and is tested to be positive. 
A5 There is no effective treatment regime for the asymptomatic individuals, and so they recover or turn symptomatic at the same rate regardless of whether they are tested positive (and hence quarantined) or not.

A graphical representation of the proposed disease propagation model is presented in Figure 1 below. The assumptions A1-A5 are quite general and concur to the observed dynamics of Covid-19 pandemic so far, even though a relatively tiny fraction of people do get infected by prolonged exposure to symptomatic patients, typically in hospitals. However, this small violation of assumption A1 is unlikely to have a significant influence on the overall dynamics, and in any case, the requisite data to account for this violation is practically unavailable. The number of reported reinfection after recovery is negligible, so are the false positive rates of both RTPCR and antigen tests (estimated to be less than 5\% [38, 39, 40, 41]). If necessary, the assumptions A2 and A3 can be generalised by adding a fraction of the recovered people in the susceptible category. Assumption A5 implies that the rate of transfer from compartment $A_{t}$ to $R_{t}^{A}$ is same as that of transfer from the compartments $Q_{t}$ to $R_{t}^{Q}$ and the rate of transfer from the compartments $A_{t}$ and $Q_{t}$ to $H_{t}$ are equal.

2.1. Disease Propagation Model. We assume an underlying Poisson process model for describing the disease dynamics. Let $\Delta C_{t}=C_{t+1}-C_{t}$ be the increments in the number of observed confirmed cases in day $t+1$. The increments $\Delta A_{t}$, etc. are defined similarly. Under our model, conditionally on the current values of different compartments (collectively denoted by $\mathcal{F}_{t}$ ), the above increments follow Poisson distributions with their mean depending on $\mathcal{F}_{t}$ and a set of rate parameter. Based on our assumptions, the evolution model are expressed as follows: 


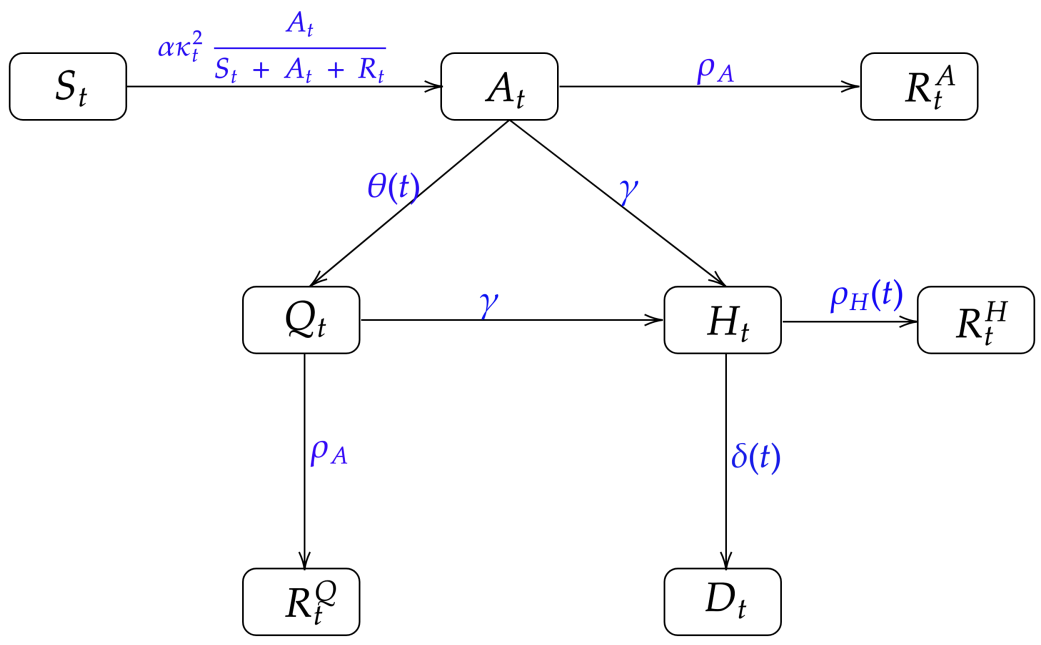

Figure 1. A graphical representation of the disease propagation model. $S_{t}, A_{t}, H_{t}, Q_{t}, D_{t}$ are the number of susceptible, infected, hospitalized, quarantined, and deceased people at time $t$ respectively. $R_{t}^{Q}, R_{t}^{H}, R_{t}^{A}$ represent the recovered population from quarantined, hospitalization, and infected but asymptotic stages respectively. The rate parameters are as described in Section 2.1.

$$
\begin{aligned}
& \mathbb{E}\left[\Delta S_{t} \mid \mathcal{F}_{t}\right]=-\left(\frac{S_{t}}{S_{t}+A_{t}+R_{t}}\right) \alpha \kappa_{t}^{2} A_{t},, \\
& \mathbb{E}\left[\Delta A_{t} \mid \mathcal{F}_{t}\right]=-\left(\theta(t)+\gamma+\rho_{A}\right) A_{t}+\left(\frac{S_{t}}{S_{t}+A_{t}+R_{t}}\right) \alpha \kappa_{t}^{2} A_{t}, \\
& \mathbb{E}\left[\Delta Q_{t} \mid \mathcal{F}_{t}\right]=\theta(t) A_{t}-\left(\gamma+\rho_{A}\right) Q_{t}, \\
& \mathbb{E}\left[\Delta H_{t} \mid \mathcal{F}_{t}\right]=\gamma\left(A_{t}+Q_{t}\right)-\left(\rho_{H}(t)+\delta(t)\right) H_{t}, \mathbb{E}\left[\Delta D_{t} \mid \mathcal{F}_{t}\right]=\delta(t) H_{t}, \\
& \mathbb{E}\left[\Delta C_{t} \mid \mathcal{F}_{t}\right]=(\theta(t)+\gamma) A_{t}, \\
& \mathbb{E}\left[\Delta R_{t}^{A} \mid \mathcal{F}_{t}\right]=\rho_{A} A_{t}, \mathbb{E}\left[\Delta R_{t}^{Q} \mid \mathcal{F}_{t}\right]=\rho_{A} Q_{t}, \mathbb{E}\left[\Delta R_{t}^{H} \mid \mathcal{F}_{t}\right]=\rho_{H}(t) H_{t}, \\
& \mathbb{E}\left[\Delta R_{t} \mid \mathcal{F}_{t}\right]=\mathbb{E}\left[\Delta R_{t}^{H} \mid \mathcal{F}_{t}\right]+\mathbb{E}\left[\Delta R_{t}^{Q} \mid \mathcal{F}_{t}\right]+\mathbb{E}\left[\Delta R_{t}^{A} \mid \mathcal{F}_{t}\right] .
\end{aligned}
$$


medRxiv preprint doi: https://doi.org/10.1101/2021.02.01.21250936; this version posted February 3, 2021. The copyright holder for this preprint

8

INFERENCE ON COVID-19 DYNAMICS

A schematic diagram of the proposed model can be found in Figure 1. All parameters in the proposed model are nonnegative. The parameter $\alpha$ is the baseline infection rate, in the absence of any social distancing. This means, $\alpha$ is the average number of susceptible individuals who may be infected on any given day by an asymptomatic but infected individual. The rate of daily recovery directly from the asymptomatic compartment is denoted by $\rho_{A}$. By assumption A5, this is also the daily rate at which a quarantined individual directly recovers. We use $\gamma$ to describe the rate at which an asymptomatic individual may become symptomatic on a given day. By assumption A5, this rate is the same whether the individual is free or in quarantine. The symbols, $\rho_{H}(t)$ and $\delta(t)$, respectively, denote is the rate at which people recover and die from the hospitalized compartment. We assume both these rates to be time-varying to reflect the changing levels of effectiveness of treatment regimes over time. We emphasize that Poisson distributions for the increments of various compartments is only a working assumption which guides our estimation strategy (e.g., by formulating appropriate transformations of variables). In Supplement Sections S6. and S7., we carry out a detailed numerical simulation under the Poisson model to validate the statistical performance of the proposed estimation procedure.

Information about daily tests is included in the model using the function $\theta(t)$. We call it the confirmed fraction $(C F)$, i.e. the fraction of currently asymptomatic individuals who are detected through testing. Parameter $\theta(t)$ would depend on the daily number of tests, as well as the efficiency of the testing strategy in identifying the infected and asymptomatic individuals. The contact tracing strategies were introduced by many states [42, 43] with varying success. In many parts of the world, people in close contact of hospitalised patients are routinely tested. This strategy is closely connected to cluster sampling, where a cluster is defined by the contacts of a hospitalised person. 

as follows:

$$
\theta(t)=\phi(t) \frac{\Delta T_{t}}{H_{t}}
$$

where $\phi(t)$ is interpreted as the testing efficiency (TE) since it measures the fraction of confirmed asymptomatic cases per test, per (currently) hospitalized patient. In Section 5 we estimate $\phi(t)$ by minimising certain loss function, from which $\theta(t)$ is subsequently estimated.

In addition, our model (see (1) ) depends on a variable $\kappa_{t}$, which is the current state of the level of interaction among individuals. Expressed as a fraction, taking value 1 for normal activity, and 0 for complete lockdown, this variable measures the prevalent social distancing in the population. In general $\kappa_{t}$ is not observable. However, often a surrogate variable based on data collected by internet service providers such as Google from the usage of smartphones [44] can be used [45, 46, 47].

From (1) the variable $\alpha \kappa_{t}^{2}$ approximately measures the daily rate at which a susceptible individual turns asymptomatic-infected. In the early stage of the epidemic, the fraction $S_{t} /\left(S_{t}+A_{t}+R_{t}\right) \approx 1$. Furthermore, rather than waiting for herd immunity to be achieved, mitigation measures are implemented in most affected places or countries to contain the spread of the disease. As a consequence, at any given time, the number of non-susceptible people is much lower as compared to the susceptible population. So $S_{t} /\left(S_{t}+A_{t}+R_{t}\right)$ has remained quite close to 1 for almost the duration of the pandemic until this point, due to the absence of mass-scale vaccination.

Notice that (5), provides a connection between the daily reported confirmed cases $\Delta C_{t}$ and the number of asymptomatic-infected individuals $A_{t}$ in the population. In our model, an asymptomatic-infected person can be discovered either through a positive test 
INFERENCE ON COVID-19 DYNAMICS

and subsequent quarantining, or through hospitalisation upon showing severe symptoms.

Therefore, once the estimates of $\theta(t)$ and $\gamma$ are available, equation(5) allows us to estimate the unknown $A_{t}$ from the observed $C_{t}$. It is also clear that, due to unavoidable severe under-reporting, $\Delta C_{t}$ will only be a fraction of the number of total infected individuals at any time point.

2.2. Some relevant epidemiological markers. The proposed model is more realistic than the traditional SIR, SEIR etc. models and allows us to estimate different epidemiological markers which can measure the dynamics of disease spread. Our focus here is on estimating epidemiological markers related to the number of asymptomatic but infected persons (i.e. $A_{t}$ ) in the population. It is well-known that the disease is mostly spread through persons in that group. Thus the proposed epidemiological markers reveal more fundamental trends of disease dynamics, than what can be obtained only by the confirmed case counts. In particular, we define the following epidemiological markers:

2.2.1. Relative Change in Confirmed Fraction ( $R C C F$ ). The relative change in confirmed fraction measures the change in the fraction of currently asymptomatic-infected individuals who are caught in the quarantine net through testing relative to the total fraction of currently infected individuals are either quarantined or hospitalised. From Section 2.1 we get:

$$
R C C F(t)=\frac{\Delta \theta(t)}{\theta(t)+\gamma}
$$

The marker $R C C F(t)$ measures the dynamics of the efficacy of the testing regime to isolate the asymptomatic but infected individuals from the population into quarantine. From (8), this marker is directly controlled by the prevalent testing strategy and efficiency. 
2.2.2. Crude infection rate (CIR) and Net Infection Rate (NIR). The crude infection rate is defined as the fraction of change in the daily confirmed cases on a day to the number of confirmed cases on that day. In our notation, it follows that:

$$
C I R(t)=\frac{\Delta^{2} C_{t}}{\Delta C_{t}}
$$

Since CIR suffers from the under-representation inherent in the reported number of confirmed cases, we define a model-based estimate for the infection rate, denoted Net Infection Rate (NIR), which is the ratio of the daily change in the number of asymptomatic-infected persons to the number of the asymptomatic-infected persons. In our notation:

$$
N I R(t)=\frac{\Delta A_{t}}{A_{t}}=\frac{C I R(t)-R C C F(t)}{1+R C C F(t)} .
$$

2.2.3. Daily New Infections (NI). From our model and assumptions, the daily number of new infections are given by the number of susceptible population who turn asymptomaticinfected on that day. From (1) we define this marker as:

$$
N I(t)=\alpha \kappa_{t}^{2}\left(\frac{S_{t}}{S_{t}+A_{t}+R_{t}}\right) A_{t}
$$

The cumulative number of new infections up to time $t$ can be defined as $C N I(t)=$ $\sum_{i=1}^{t} N I(i)$

2.2.4. Doubling Times and Rates: The doubling time at time $t$, denoted $t_{d}(t)$ measures how much longer it would take for the number of infected upto time $t$ to double. The doubling rate at time $t, \tilde{\xi}(t)$ is given by the inverse of the doubling time. A higher doubling rate reflects the faster spread of infection. This rate is often used to measure the effect of social distancing campaigns, improved hygiene and case tracking. 
The doubling time for $C_{t}$ computed using the relationship $C_{t+t_{d}(t)} / C_{t}=2$. It can be shown (see the Supplement Section S4.) $t_{d}(t)=\left[\frac{d}{d t} \log C_{t}\right]^{-1}$. That is the doubling rate $\tilde{\xi}(t)=t_{d}(t)^{-1}=\frac{d}{d t} \log C_{t}$. Doubling rates for other compartments can be computed similarly.

2.2.5. Crude and Net Case Fatality Rates. In general a case fatality rate at time $t$ is given by the ratio of the total death count and the total case count at that time. Depending on whether the reported case counts or the actual case counts are used, we can define two different case fatality rates. The crude case fatality rate $(\mathrm{CFR})$ is defined as:

$$
C F R(t)=\frac{D_{t}}{C_{t}} \times 100
$$

whereas the net case fatality rate is given by

$$
N F R(t)=\frac{D_{t}}{C N I(t)} \times 100
$$

2.2.6. The Basic Reproduction Rate. In the conventional SIR or SIER models, basic reproduction rate $\left(R_{0}\right)$, which measures the expected number of cases directly generated by one case in a population where all individuals are susceptible to infection [48] is used to determine the nature and rate of growth of the pandemic. Unlike these models, our model is more detailed and allows for time varying parameters and as a result, the conventional $R_{0}$ cannot be directly estimated from our model. The closest epidemiological quantity we can observe is the background infection rate, $\alpha$, measuring the average number of susceptible individuals who may be infected on any given day by an asymptomatic but infected individual. However, by following the next generation method [49, 50] an analogue of the basic reproduction rate for the compartment $A_{t}$ can be computed. 
By focusing on the compartment $A_{t}$, under our assumptions from (2) new infections arrive at the compartment at the rate of $\alpha \kappa_{t}^{2} S_{t} /\left(S_{t}+A_{t}+R_{t}\right)$ and leave at the rate of $\left(\theta(t)+\gamma+\rho_{A}\right)$. There is no other pathway for disease spread. Thus we can define an analogue of the basic reproduction rate as:

$$
\tilde{R}_{0}(t)=\frac{\alpha \kappa_{t}^{2}}{\theta(t)+\gamma+\rho_{A}}\left(\frac{S_{t}}{S_{t}+A_{t}+R_{t}}\right)
$$

Note that, the proposed $\tilde{R}_{0}(t)$ can be interpreted in the same way as the conventional basic reproduction rate. By construction $\tilde{R}_{0}(t)<1$ indicates negative growth of the number of asymptomatic-infected persons, whereas $\tilde{R}_{0}(t)>1$ indicates its positive growth. However, temporal variation of $\tilde{R}_{0}(t)$ is more complex. Assuming that, $S_{t} /\left(S_{t}+A_{t}+R_{t}\right) \approx 1, \tilde{R}_{0}$ can decrease with time either due to reduction in $\kappa_{t}$, that is the current state of interaction among individuals, or due to an increase in the confirmed fraction $\theta(t)$. That is, the proposed $\tilde{R}_{0}(t)$ is directly influenced by the mitigation efforts such as social distancing, adherence to use of masks, increased testing and subsequent quarantining, hospitalisation of symptomatic patients etc.

Most epidemiological models such as SIR, SIER etc., assume fixed doubling rate parameters. In reality, however, the doubling time is a dynamic quantity, which changes continuously due to mitigation efforts and the inherently changing nature of virus-spreading mechanisms. It is then vital that policymakers and researchers have access to frequent and up-to-date estimates of doubling time [51]. For example, [52] provided early, fixed-in-time estimates of epidemic parameters of COVID-19 (e.g. growth rate, doubling time, basic reproduction number, case detection rate) during the first 50 days of onset in China. In recent work [53, 54] the basic reproduction number and doubling time have been studied in a dynamic manner by considering a varying coefficient model with daily new cases 
medRxiv preprint doi: https://doi.org/10.1101/2021.02.01.21250936; this version posted February 3, 2021. The copyright holder for this preprint (which was not certified by peer review) is the author/funder, who has granted medRxiv a license to display the preprint in perpetuity.

It is made available under a CC-BY-NC-ND 4.0 International license .

14

INFERENCE ON COVID-19 DYNAMICS

as the response and time as a predictor. A related approach focused on the real-time estimation of case fatality rates using Poisson mixture models can be found in [55].

\section{Results : Applichtion to COVID-19 DAta from the USA}

3.1. Data Preparation. We consider the dynamics of the spread of COVID-19 in various states of USA for a tentative time window of late April to mid December. The proposed model is based on the observed state-wise daily counts of confirmed infections, deaths, hospitalisations and reported recoveries from the hospitals and quarantining facilities. Daily counts of the confirmed COVID-19 cases in various states were obtained from the COVID-19 Data Repository maintained by the Center for Systems Science and Engineering (CSSE) at Johns Hopkins University. This is publicly available at https://github.com/CSSEGISandData/COVID-19 and was accessed on December 15, 2020. The state-wise daily counts of positive and negative COVID-19 test results, current hospitalization, and recovery per day and state, were obtained from the CDC data repository -the COVID Tracking Project and are publicly available at https://COVIDtracking.com/ (accessed on December 15, 2020.)

The collected noisy data used is pre-processed and cleaned, removing the irregularities present in the recording and maintenance of the data repositories. Any missing or evidently wrong (e.g. negative counts) observations were replaced by the average of the data from adjacent five days. Inherent noise present in the daily counts was removed by pre-smoothing the trajectories using a Lowess method [56, 57, 58, 59] with bandwidth $1 / 16$.

3.2. Results. Unfortunately, a continuous record on hospitalization and recovery information were not available for many states. For example, most counties in California are not reporting recovery information. Data on Hospitalization is found to be updated once 
medRxiv preprint doi: https://doi.org/10.1101/2021.02.01.21250936; this version posted February 3, 2021. The copyright holder for this preprint a week in Massachusetts and Florida. New York on the other hand started documenting the hospitalization information only after the initial surge of the pandemic was over for the state. In our analysis we only consider the states for which daily observations on $C_{t}$, $D_{t}, R_{t}^{\text {reported }}, Q_{t}$, and $H_{t}$ are available throughout the time window under consideration. Any missing/negative values are replaced by the average of the adjacent five days' data. For a few states e.g. Alabama, the available data turned out to be too unreliable. We present results for fifteen states in US which demonstrate the efficacy of the proposed model and the estimation methods. For succinct representation, the results from only one state i.e. Utah is presented in details below. The results for other fourteen states can be found in the Supplement Section S9.

3.2.1. Case study for the state Utah. We present our results for the state Utah for the time window between 7th May, 2020 to 4th December, 2020. The time interval includes the Thanksgiving weekend (27th -28th November, 2020), when due to the long holiday, the reported data may be unreliable. In Figure 2 plots of various time-varying compartments and epidemiological markers defined in Section 2.2. The plots of the parameters with their residual bootstrap confidence intervals can be found in Figure 4. Due to unreliable reporting around the Thanksgiving holiday, the estimates values after 21st November, 2020 should be interpreted with caution.

The curves in Figure 2(a) compare the observed and the fitted number of daily number of people in the hospitals. It can be seen that, the fitted values obtained from the model closely follow the observed values. This validates our proposed model and the estimation procedure. From the data and the fit two waves of infection can be identified. It seems the first wave starts at the end of May, 2020 stabilises and begins to die down around 7th August, 2020. The daily number of people in hospitals starts increasing again around the end of August, 2020. 
16

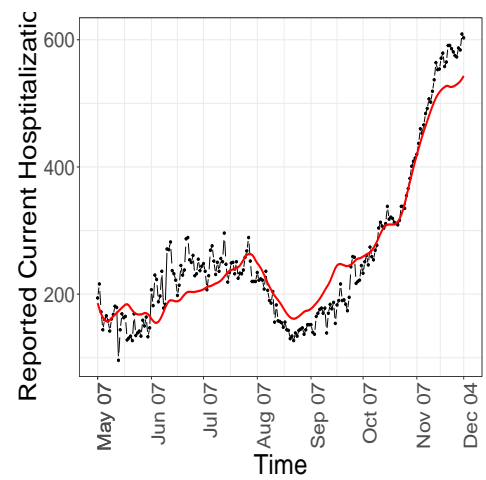

(a) Daily Hospitalisations, observed (-), fitted (-)

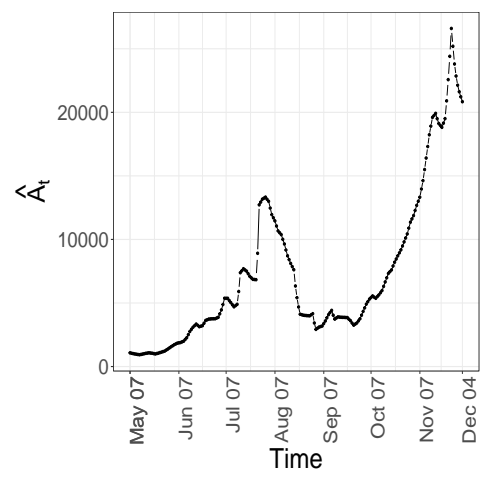

(d) $\hat{A}_{t}$

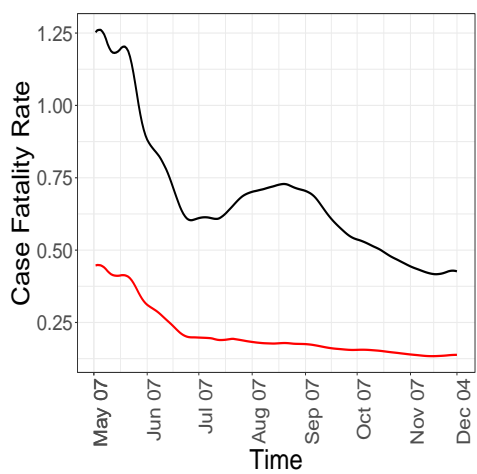

(g) CFR (-) and NFR (-)

INFERENCE ON COVID-19 DYNAMICS

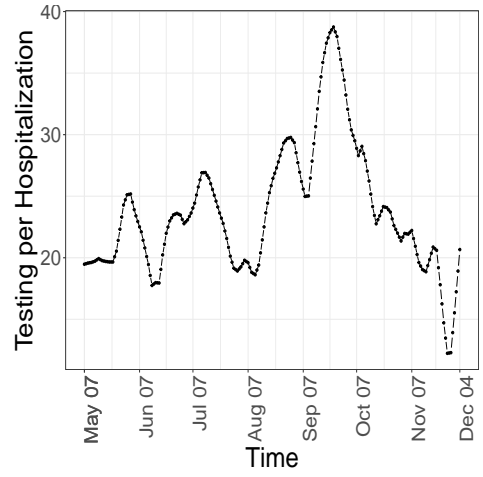

(b) $\Delta T_{t} / H_{t}$

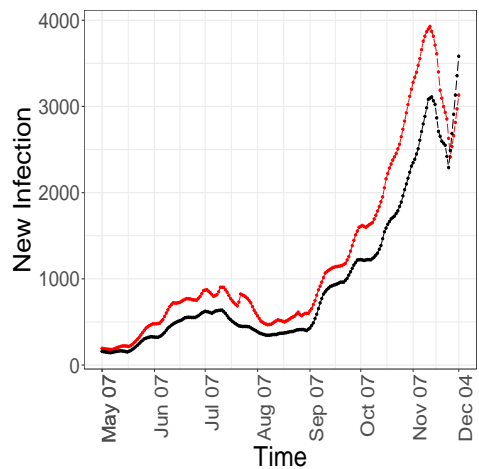

(e)

Daily New Infections, $\Delta C_{t}(-)$ and $N I(-)$

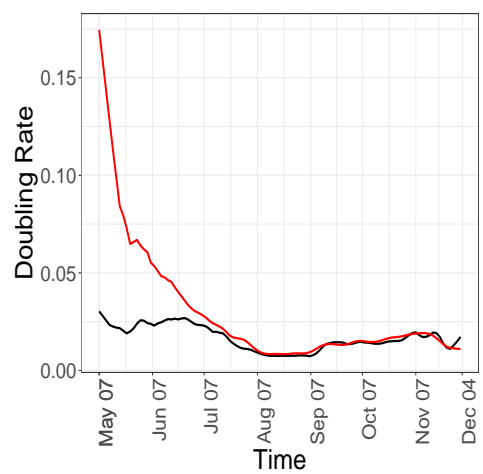

(h) Doubling rate, $C_{t}(-)$ and $C N I(-)$

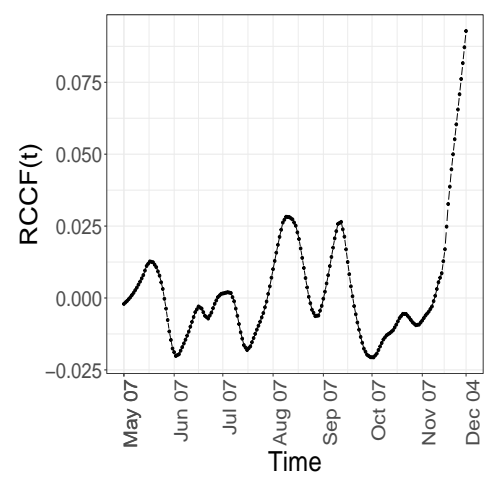

(c) RCCF

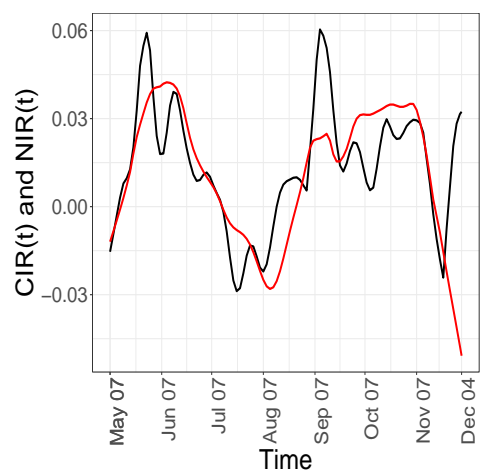

(f) CIR (-) and NIR (-)

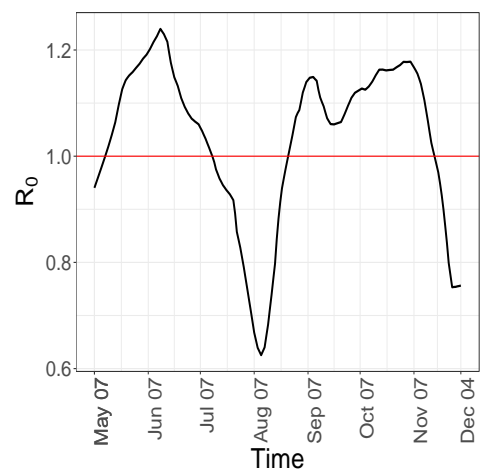

(i) $\tilde{R}_{0}$

FiguRE 2. Temporal patterns of some compartments and epidemiological markers for Utah. 


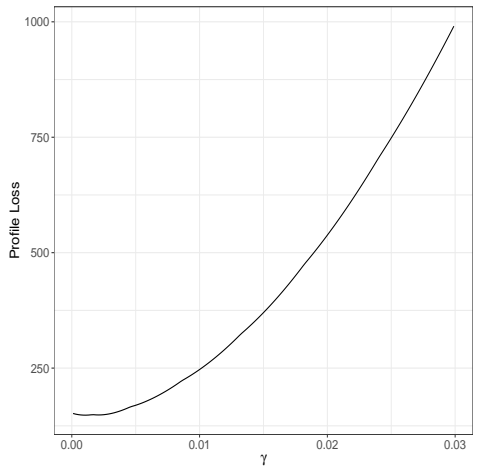

(a) $\gamma-\operatorname{vs}-L^{h}\left(\gamma, \rho_{A}\right)$

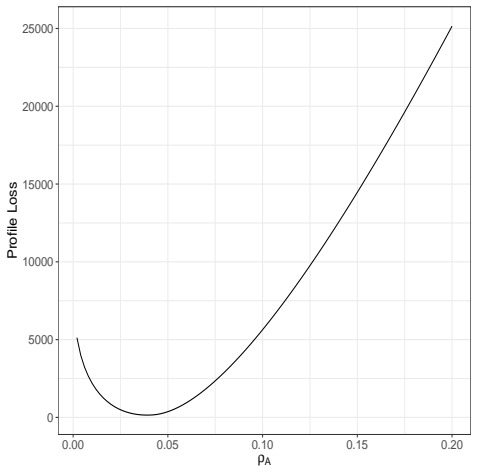

(b) $\rho_{A}$-vs- $L^{h}\left(\gamma, \rho_{A}\right)$

FiguRE 3. The profile loss as a function of $\gamma$ and $\rho_{A}$ respectively.

The estimated number of infected asymptomatic people (Figure $2(\mathrm{~d})$ ) shows a similar pattern. From a high point around the beginning of August it dips to a low value at the end of August. The number remains stable for a few weeks and starts growing again at the end of September. The two waves can also be clearly observed from the plot of the proposed analogue of the basic reproduction rate $\tilde{R}_{0}$ in Figure 2(i), In the time period under consideration, the estimated $\tilde{R}_{0}$ was larger than one in two sub-intervals, namely from middle May to middle of July and then from end of August to beginning of November. The plot of the number of daily new and daily reported infections (Figure 2(e) shows a local maxima near the middle of November. However, we cannot rule out the boundary effect as its cause.

The plots of CIR and NIR seem to be similar (Figure 2(f)]. In fact, the observed doubling rate obtained from $C_{t}$ and that estimated from $C N I$ seems to be very close in the second wave of the pandemic (see Figure 2(h)). This implies that in the second wave the reporting kept pace with the spread of the disease. Figure $2(\mathrm{~g})$ shows the crude and net fatality rates. Due to the denominator effect, naturally the crude fatality rate is much faster than the net fatality rate. However, our estimate of NFR is mostly below $0.25 \%$, which complies with widely held beliefs [60, 24, 61, 62]. 
medRxiv preprint doi: https://doi.org/10.1101/2021.02.01.21250936; this version posted February 3, 2021. The copyright holder for this preprint

18

INFERENCE ON COVID-19 DYNAMICS

The estimate of $\delta(t)$ in Figure $4(\mathrm{~d})$ seems to remain stable throughout the time period under consideration. The $\widehat{\rho_{H}}(t)$ shows an overall increasing trend. On the other hand, The estimate of $\theta(t)$ decreases to a near zero value at the end of the first wave (7th August, 2020) it then increases to its maximum value at the end of September and starts to decrease again. The parameters $\left(\gamma, \rho_{A}\right)$ are estimated based on minimization of the profile loss using a grid search algorithm with grid size 0.0001. In Figures 4(a) and 4(b) the estimates from residual bootstrap samples take discrete values, resulting in a discrete histogram counts.

The daily number of tests and its effect in quarantining asymptomatic but infected people can be judged from the Figures 2(b) and 2(c), The state of Utah increased its testing capacity by public-private partnership. Empirical comparison of the Figures 2(a) and 2(b) seems to reveal that although the number of daily tests could keep pace with daily number of hospitalised patients up to the third week of September, but growing number of hospitalised people ultimately outpaced the number of daily tests. Note that, estimated $\theta(t)$ increases at the onset of the second wave (see Figure 4(c) between 7th, August and 21st, September), however, from figure 2(d), $\hat{A}_{t}$ remains more or less constant. Thus, growth in the number of new infections could be due to the increase in $\kappa_{t}$, that is due to more interaction among individuals and less social distancing.

3.2.2. Summary of Results for Other States. We present a summary of the results obtained from applications of proposed method on the data procured from fifteen other states in

\begin{tabular}{c||c|c|c|c}
\hline & Estimate & $95 \%$ Confidence Interval & Mean & s.d. \\
\hline \hline$\gamma$ & 0.0011 & {$[0.0011,0.0023]$} & 0.0017 & 0.0003 \\
\hline$\rho_{A}$ & 0.0400 & {$[0.0380,0.0400]$} & 0.0386 & 0.0010 \\
\hline
\end{tabular}

TABlE 1. Estimates, and the residual bootstrap Confidence intervals, mean and standard deviations for the time-invariant parameters for Utah. The latter three are computed based on 1000 bootstrap resamples. 


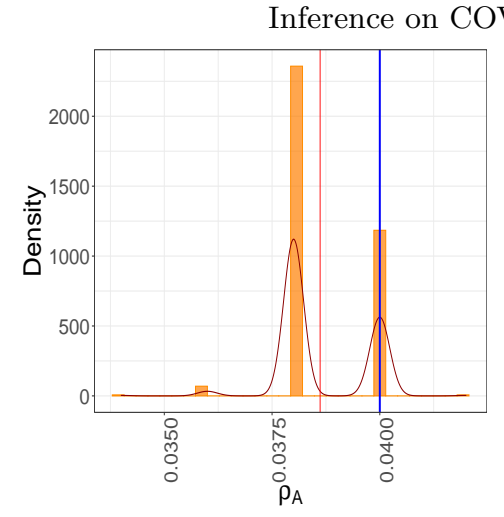

(a) $\rho_{A}$,

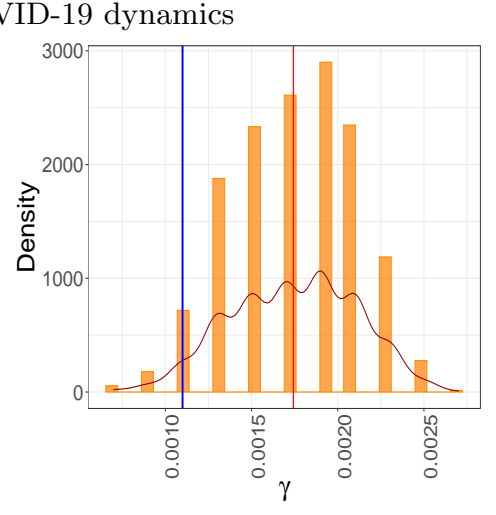

(b) $\gamma$,

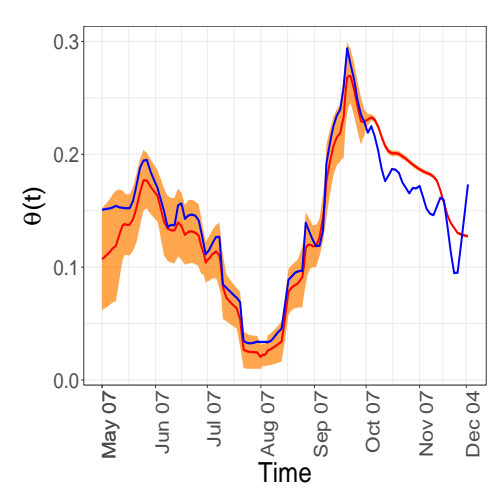

(c) $\theta(t)$

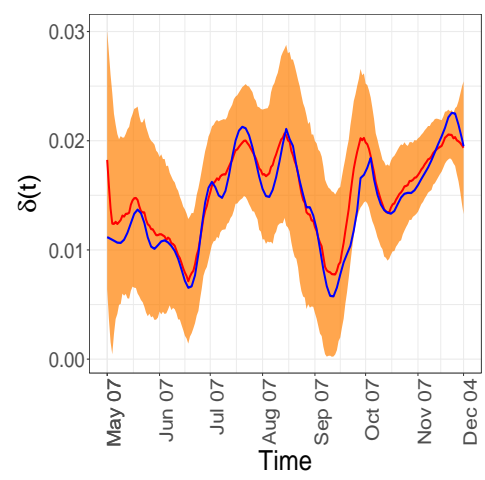

(d) $\delta(t)$

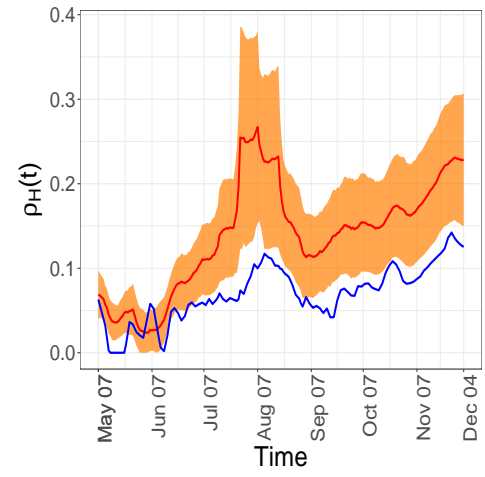

(e) $\rho_{H}(t)$

FiguRE 4. Estimates and residual bootstrap based confidence intervals for time invariant and time-varying parameters for the state of Utah. The estimate from the data is in blue. The $95 \%$ confidence band is in yellow and the mean of the bootstrap estimates are presented in red.

USA. The estimated parameters are in Table 2. The time-varying parameters, $(\phi(t)$, $\left.\rho_{H}(t), \delta(t)\right)$, are summarised by their means. The computed $\hat{\gamma}$, that is, the rate for an asymptomatic person turning symptomatic on a particular day is the smallest in Arizona and largest in Tennessee. This estimate is smaller than 0.001 for Arizona and Idaho. Minnesota, has by far the highest recovery rate for an asymptomatic person without needing hospitalization on a particular day (i.e. $\widehat{\rho}_{A}$ ). For Iowa, Nebraska, Pennsylvania, and Utah this rate is comparable and reasonably high, whereas Arizona, Delaware and Idaho have their $\widehat{\rho}_{A}$ value below 0.01 . The average confirmed fraction $\overline{\hat{\theta}}$ is larger than 


\begin{tabular}{c||c|c|c|c|c}
\multicolumn{7}{c|}{ INFERENCE ON COVID-19 DYNAMICS } \\
\hline & $\hat{\gamma}$ & $\hat{\rho}_{A}$ & $\widehat{\delta}(t)$ & $\widehat{\widehat{\rho}}_{H}(t)$ & $\widehat{\theta}(t)$ \\
\hline \hline Arizona & 0.0003 & 0.002 & 0.0208 & 0.0023 & 0.0887 \\
\hline Arkansas & 0.0029 & 0.094 & 0.0249 & 0.0975 & 0.0809 \\
\hline Delaware & 0.0017 & 0.008 & 0.0159 & 0.0093 & 0.1076 \\
\hline Idaho & 0.0009 & 0.010 & 0.0230 & 0.0138 & 0.0289 \\
\hline Iowa & 0.0011 & 0.032 & 0.0263 & 0.0372 & 0.0478 \\
\hline Minnesota & 0.0023 & 0.128 & 0.0315 & 0.0654 & 0.0899 \\
\hline Nebraska & 0.0011 & 0.020 & 0.0141 & 0.0266 & 0.0394 \\
\hline Ohio & 0.0023 & 0.048 & 0.0180 & 0.0532 & 0.0625 \\
\hline Oklahoma & 0.0037 & 0.084 & 0.0122 & 0.1029 & 0.0494 \\
\hline Pennsylvania & 0.0013 & 0.026 & 0.0293 & 0.0372 & 0.0535 \\
\hline South Dakota & 0.0021 & 0.058 & 0.0190 & 0.0922 & 0.0262 \\
\hline Tennessee & 0.0059 & 0.064 & 0.0158 & 0.0413 & 0.1206 \\
\hline Texas & 0.0019 & 0.036 & 0.0207 & 0.0341 & 0.0212 \\
\hline Utah & 0.0011 & 0.040 & 0.0144 & 0.0252 & 0.1434 \\
\hline Wisconsin & 0.0017 & 0.068 & 0.0217 & 0.0707 & 0.0477 \\
\hline 2.
\end{tabular}

TABLE 2. Mean estimated parameters for different states in the USA.

0.1 in Delaware, Tennessee and Utah. It is lowest in Texas. This can be associated with better estimates obtained for these states due to the availability of more reliable data, whereas for Idaho, South Dakota, and Texas, a lower value of there epi-markers tend to give evidence for a more relaxed testing paradigm. More testing is required for isolating the confirmed cases to contain the disease faster, which can be reflected in the numbers for these states. The detailed results and bootstrap confidence regions for these additional states can be found in the Supplement Section S9.

Among the states not included in Table 2, many, such as California did not report all the required compartments. For many states such Alabama, Colorado, Maryland, Massachusetts, North Carolina etc. the reported data produced monotone profile likelihoods which yielded unreliable boundary estimates. This could be due to the change in definition of many compartments over time, which violated our assumptions. Furthermore, for some states such as New York, New Jersey, Michigan etc., the pandemic started quite early and ran its course even before a proper testing protocol and other mitigation measures could 
be introduced. Thus the data from these states is contaminated with an inherent bias, the number of people in quarantine or symptomatic states are to too low to produce reliable estimates.

\section{Discussion}

We introduce a multi-compartment model for COVID-19 dynamics which can incorporate data from compartments like quarantine, hospitalisation etc. Unlike the conventional SIR and similar models, the proposed model is based on interpretable time-varying parameters, which are more suitable for describing the disease dynamics in the presence of mitigating procedures. It also incorporates the information about testing and subsequent quarantining. We estimate the model parameters using profile likelihood and nonparametric regression. This provides a much faster alternative to Markov Chain Monte Carlo based Bayesian models which are commonly used in estimating SIR parameters. Using the proposed detailed and robust model one can estimate the daily number of asymptomatic but infected individuals, who are universally regarded as the key agent for the COVID-19 spread. We define several epidemiological markers which uses the number of asymptomatic-infected individuals and therefore reveal the true underlying dynamics of the pandemic.

Our model only uses information on the number of confirmed infected, hospitalised, deaths and total reported recoveries from hospitals and the quarantine. We don't require those numbers separately. However, such numbers are often available. In such a case, the loss function in (21) can be simplified a bit. The details can be found in the Supplement in Section S2.

The model parameters have been estimated assuming that no information about the mobility within the population is available. Such information identifies the parameters 
medRxiv preprint doi: https://doi.org/10.1101/2021.02.01.21250936; this version posted February 3, 2021. The copyright holder for this preprint

$22 \quad$ INFERENCE ON COVID-19 DYNAMICS

$\kappa_{t}$ and $\alpha$ in our model. Reliable data on the compliance to social distancing, mask wearing etc. are difficult to get. Various aspects of the mobility data available from Google can be one potential surrogate for $\kappa_{t}$ [63, 64]. However, such data only look into the fraction of people going to workplace or recreation and so on, and does not collect information on the people who are the super spreaders or not wearing masks. Thus, it does not necessarily reflect the the social mobility index $\kappa_{t}$, as incorporated in our model. In the Supplement (see Sections S3. and S9.), we present results by using the Google mobility data as a surrogate to $\kappa_{t}$. In particular, information on the change in the mobility patterns, as the percentage decrease (increase) from the baseline, in different areas such as parks, residential locations, retail locations, among others during the pandemic from the Google mobility database were obtained. The publicly available data was sourced from https://www.google.com/COVID19/mobility/ (accessed on December 15, 2020). When information on $\kappa_{t}$ is available, the parameter $\alpha$, which is the average number of susceptible individuals who may be infected in a day by an asymptomatic-infected individual is identifiable and can be estimated. The details can be found in the Supplement Section S3.

The proposed method and estimation procedure do not explicitly use the underlying assumption of a Poisson process. In the Supplement (see Section S6.- S8.), however, we use an ensemble of independent Poisson processes to simulate data from the proposed model. These aggregated data sets are then used to accurately estimate various parameters, which validate our estimation procedure. The aggregation has the effect of increasing the number of observations in the compartments and thereby improving estimation accuracy. If the number of individuals in the symptomatic or quarantined compartments are low, e.g. at the onset of the pandemic, inherent biases are introduced in the estimated trajectories. A bigger sample size is required to correct such contaminants. 
It should be noted that COVID-19 analyses based on published case and death counts, including those conducted here, are subject to the same biases which affect the accuracy of the data, primarily due to under-reporting [65] or mis-recording of the data, the degree of which varies by country [66]. The reasons for such under-reporting are many, including insufficient testing materials, political incentives, and administrative delays. Furthermore, our model assumes a closed population. It ignores migration between cities, states or countries which play an essential role in the propagation of the disease. We only count the deaths solely due to Covid-19 infections and as such completely ignore any competing causes of morbidity, as well as increase in population due to new births.

With this caveat in mind, the study of available data presented in this article nevertheless provides useful insights into the COVID-19 propagation and ways to control it. It clearly follows that in order to break the chain of transmission and "flatten the curve", we need extensive testing and adhere to strict social distancing protocols.

\section{Methods : Parameter And COMPartment Estimation}

The core of our estimation strategy is to utilize (1)-(7) to formulate appropriate regression problems. Our estimation procedure is based on the availability of the compartments $C_{t}, D_{t}, H_{t}, Q_{t}, T_{t}$ and $R_{t}^{\text {reported }}$ only. We do not assume that data on the social distancing factor $\kappa_{t}$ is available. Described crudely, the proposed estimation method uses local regression (linear or nonlinear) methods for estimating the time-varying parameters, while profiling over the time-independent ones.

In the absence of data on $\kappa_{t}$, the parameter $\alpha$ in (1) is not identifiable. We first describe how the product $\alpha \kappa_{t}^{2}$ can be estimated. Notice that, ignoring the stochasticity, we may rewrite (5) as

$$
\Delta C_{t}=(\theta(t)+\gamma) A_{t}
$$


Defining $\eta(t)=\theta(t)+\gamma$, and applying the difference operator on both sides of (16), and finally dividing both sides by $\Delta C_{t}$, we obtain

$$
\frac{\Delta^{2} C_{t}}{\Delta C_{t}}=\left(1+\frac{\Delta \eta(t)}{\eta(t)}\right) \frac{\Delta A_{t}}{A_{t}}+\frac{\Delta \eta(t)}{\eta(t)} .
$$

Now, ignoring the second order factor $\left(\Delta \eta(t) \Delta A_{t}\right) /\left(\eta(t) A_{t}\right)$, from (2), at the onset of the epidemic (i.e. $S_{t} /\left(S_{t}+A_{t}+R_{t}\right) \approx 1$ ), we have the approximate relationship:

$$
\frac{\Delta^{2} C_{t}}{\Delta C_{t}} \approx \frac{\Delta \eta(t)}{\eta(t)}-\eta(t)-\rho_{A}+\alpha \kappa_{t}^{2} .
$$

Note that (18) establishes an approximate linear relationship, between the observable quantity $\Delta^{2} C_{t} / \Delta C_{t}$ and the product $\alpha \kappa_{t}^{2}$. Below we show that, the other parameters in (18) can be estimated, from the available data. These estimates can be plugged in to get an estimate of $\alpha \kappa_{t}^{2}$.

5.1. Point Estimates. Broadly speaking, the estimation strategy consists of separating the time-dependent and time-independent parameters, into vectors $\boldsymbol{\beta}_{t}=\left(\phi(t), \rho_{H}(t), \delta(t)\right)$ and $\boldsymbol{\zeta}=\left(\gamma, \rho_{A}\right)$ respectively. First the vector $\boldsymbol{\zeta}$ is kept fixed and for each $t$ the time dependent parameter $\boldsymbol{\beta}_{t}$ is estimated (denoted $\widehat{\boldsymbol{\beta}}_{t}^{h}(\boldsymbol{\zeta})$ ) by minimizing the "conditional" local loss function $\widetilde{L}_{t}^{h}\left(\boldsymbol{\beta}_{t} \mid \boldsymbol{\zeta}\right)$ (described below) with respect to $\boldsymbol{\beta}_{t}$, subject to appropriate constraints on the parameters (non-negativity as well as certain upper bounds). The optimal local conditional loss is then combined across different time points to obtain the profile loss function for $\boldsymbol{\zeta}$, which is given by

$$
L^{h}(\boldsymbol{\zeta})=\sum_{t} \widetilde{L}_{t}^{h}\left(\widehat{\boldsymbol{\beta}}_{t}^{h}(\boldsymbol{\zeta}) \mid \boldsymbol{\zeta}\right)
$$

The estimate $\widehat{\boldsymbol{\zeta}}^{h}$ of $\boldsymbol{\zeta}$ is obtained by minimizing $L^{h}(\boldsymbol{\zeta})$ under appropriate constraints. We update the estimates of $\boldsymbol{\beta}_{t}$ as $\widehat{\boldsymbol{\beta}}_{t}^{h}=\left(\widehat{\phi}(t), \widehat{\rho}_{H}(t), \widehat{\delta}(t)\right)=\widehat{\boldsymbol{\beta}}_{t}^{h}\left(\widehat{\boldsymbol{\zeta}}^{h}\right)$. 
In order to define the conditional loss function, let $K(\cdot)$ be a nonnegative kernel integrating to one. Now, for a bandwidth parameter $h>0$, the local weighted conditional loss function of $\boldsymbol{\beta}_{t}$, given $\boldsymbol{\zeta}$ is defined as:

$$
\widetilde{L}_{t}^{h}\left(\boldsymbol{\beta}_{t} \mid \boldsymbol{\zeta}\right)=\sum_{s} \frac{1}{h} K\left(\frac{t-s}{h}\right) d_{s}\left(\boldsymbol{\beta}_{t} \mid \boldsymbol{\zeta}\right)
$$

where

$$
\begin{aligned}
d_{s}\left(\boldsymbol{\beta}_{t} \mid \boldsymbol{\zeta}\right)= & \left|\sqrt{\Delta H_{s}+\Delta D_{s}+\Delta R_{s}^{\text {reported }}}-\sqrt{\left(\rho_{A}+\gamma\right) Q_{s}+\frac{\gamma \Delta C_{s}}{\phi(t) F_{s}+\gamma}}\right|^{2} \\
& +\left|\sqrt{\Delta R_{s}^{\text {reported }}}-\sqrt{\rho_{A} Q_{s}+\rho_{H}(t) H_{s}}\right|^{2}+\left|\sqrt{\Delta D_{s}}-\sqrt{\delta(t) H_{s}}\right|^{2} .
\end{aligned}
$$

Note that the RHS of (21) only uses the observed data. The first addendum originates from equations (4), (5) and (6). The second and the third term use equations (6) and (4) respectively. The square-root transformation of the responses are used as a variance stabilising transformation, which is driven by the assumed Poissonian characteristics of the responses. Also by construction, the estimate of $\delta(t)$ does not depend on $\boldsymbol{\zeta}$.

Estimated values of the parameters readily yields estimates of the key compartments of the model. In particular, from the definition of $\theta(t),(16)$ and $(17)$ we get:

$$
\widehat{\theta}(t)=\widehat{\phi}(t) \frac{\Delta T_{t}}{H_{t}}, \quad \hat{A}_{t}=\frac{\Delta C_{t}}{\widehat{\theta}_{t}+\hat{\gamma}}, \quad \widehat{\left(\frac{\Delta A_{t}}{A_{t}}\right)}=\frac{\frac{\Delta^{2} C_{t}}{\Delta C_{t}}-\frac{\Delta \hat{\theta}(t)}{\hat{\theta}(t)+\hat{\gamma}}}{1+\frac{\Delta \hat{\theta}(t)}{\hat{\theta}(t)+\hat{\gamma}}}, \quad \widehat{\Delta A_{t}}=\widehat{\left(\frac{\Delta A_{t}}{A_{t}}\right)} \hat{A}_{t} .
$$

Now, by plugging in $\hat{\gamma}, \widehat{\theta}(t), \hat{A}_{t}$ and $\hat{\delta}(t)$ in (4) we get an updated estimator of $\rho_{H}(t)$ as

$$
\widehat{\rho}_{H}(t)=\frac{\Delta H_{t}-\hat{\gamma}\left(\hat{A}_{t}+Q_{t}\right)+\hat{\delta}(t) H_{t}}{H_{t}}
$$


INFERENCE ON COVID-19 DYNAMICS

Finally, using (17) an estimate of $\alpha \kappa_{t}^{2}$ can be obtained as:

$$
\widehat{\alpha \kappa_{t}^{2}}=\widehat{\left(\frac{\Delta A_{t}}{A_{t}}\right)}+\hat{\theta}(t)+\hat{\gamma}+\hat{\rho}_{A} \text {. }
$$

The rest of the compartments can be estimated by plugging in the appropriate parameter or compartment estimates in equations (1) - (7) (see the Supplement Sections S1. and S3.).

The tuning parameter $h$ in 20 is obtained by minimising a standardised $L_{1}$ distance between the fitted and model based estimates of various compartments through a crossvalidation strategy. The actual minimisation is achieved by a grid-search. Details can be found in the Supplement Sections S1. and S2.

5.2. Confidence Intervals. We employ residual bootstrap [67, 68, 69] to compute the confidence intervals for our parameter and compartment estimates. Briefly put, the technique adds resampled residuals to the fitted values to create several "resampled" datasets. The point estimation technique described above is applied to each of these resampled datasets to create a new set of parameter and compartment estimates. The empirical distribution of these estimates are then used to construct the confidence interval. The details of the algorithm can be found in the Supplement Section S5. The theoretical validity of the residual bootstrap method is well justified in existing literature [70, 71].

\section{Data And Code Availability}

All data necessary for the replication of our results is collated in https://github. com/Satarupa3671/COVID-19-Nonparametric-Inference. The data for the number of COVID cases, deaths, hospitalizations and recovery were originally collected from https: //covidtracking.com/data/download while the social mobility data was sourced from https://www.google.com/covid19/mobility. 
medRxiv preprint doi: https://doi.org/10.1101/2021.02.01.21250936; this version posted February 3, 2021. The copyright holder for this preprint (which was not certified by peer review) is the author/funder, who has granted medRxiv a license to display the preprint in perpetuity. It is made available under a CC-BY-NC-ND 4.0 International license .

Inference on COVID-19 dynamics
All code necessary for the replication of our results is collated in https://github.com/

Satarupa3671/COVID-19-Nonparametric-Inference.

\section{REFERENCES}

[1] Johns Hopkins University. Global cases by the center for systems science and engineering (CSSE) at Johns Hopkins University. Coronavirus Resource Center, 2020.

[2] Markus Hoffmann et al. SARS-CoV-2 cell entry depends on ACE2 and TMPRSS2 and is blocked by a clinically proven protease inhibitor. Cell, 2020.

[3] Barak Mizrahi, Smadar Shilo, Hagai Rossman, Nir Kalkstein, Karni Marcus, Yael Barer, Ayya Keshet, Na'ama Shamir-Stein, Varda Shalev, Anat Ekka Zohar, et al. Longitudinal symptom dynamics of covid-19 infection. Nature Communications, 11(1):1-10, 2020.

[4] H Juliette T Unwin, Swapnil Mishra, Valerie C Bradley, Axel Gandy, Thomas A Mellan, Helen Coupland, Jonathan Ish-Horowicz, Michaela AC Vollmer, Charles Whittaker, Sarah L Filippi, et al. State-level tracking of covid-19 in the united states. Nature Communications, 11(1):1-9, 2020.

[5] Alberto Godio, Francesca Pace, and Andrea Vergnano. Seir modeling of the italian epidemic of sars-cov-2 using computational swarm intelligence. International Journal Of Environmental Research And Public Health, 17(10):3535, 2020.

[6] Oyungerel Byambasuren, Magnolia Cardona, Katy Bell, Justin Clark, Mary-Louise McLaws, and Paul Glasziou. Estimating the extent of asymptomatic covid-19 and its potential for community transmission: systematic review and meta-analysis. Official Journal Of The Association Of Medical Microbiology And Infectious Disease Canada, $5(4): 223-234,2020$. 
28 INFERENCE ON COVID-19 DYNAMICS

[7] Lili Wang, Yiwang Zhou, Jie He, Bin Zhu, Fei Wang, Lu Tang, Michael Kleinsasser, Daniel Barker, Marisa C Eisenberg, and Peter XK Song. An epidemiological forecast model and software assessing interventions on the covid-19 epidemic in china. Journal Of Data Science, 18(3):409-432, 2020.

[8] Yuanji Tang and Shixia Wang. Mathematic modeling of covid-19 in the united states. Emerging Microbes \& Infections, 9(1):827-829, 2020.

[9] Jin Wang. Mathematical models for covid-19: applications, limitations, and potentials. Journal Of Public Health And Emergency, 4, 2020.

[10] Youssoufa Mohamadou, Aminou Halidou, and Pascalin Tiam Kapen. A review of mathematical modeling, artificial intelligence and datasets used in the study, prediction and management of covid-19. Applied Intelligence, 50(11):3913-3925, 2020.

[11] Giorgio Kaniadakis, Mauro M Baldi, Thomas S Deisboeck, Giulia Grisolia, Dionissios T Hristopulos, Antonio M Scarfone, Amelia Sparavigna, Tatsuaki Wada, and Umberto Lucia. The $\kappa$-statistics approach to epidemiology. Scientific Reports, 10(1):1$14,2020$.

[12] Faical Ndairou, Ivan Area, Juan J Nieto, and Delfim FM Torres. Mathematical modeling of covid-19 transmission dynamics with a case study of wuhan. Chaos, Solitons \& Fractals, page 109846, 2020.

[13] Yuanyuan Dong, Xi Mo, Yabin Hu, Xin Qi, Fan Jiang, Zhongyi Jiang, and Shilu Tong. Epidemiology of covid-19 among children in china. Pediatrics, 145(6), 2020.

[14] Kenneth McIntosh, MS Hirsch, and A Bloom. Coronavirus disease 2019 (covid-19): Epidemiology, virology, and prevention. Lancet. Infect. Dis, 1:2019-2020, 2020.

[15] Tobias S Brett and Pejman Rohani. Transmission dynamics reveal the impracticality of covid-19 herd immunity strategies. Proceedings of the National Academy of Sciences, 117(41):25897-25903, 2020. 
[16] Andrea L Bertozzi, Elisa Franco, George Mohler, Martin B Short, and Daniel Sledge. The challenges of modeling and forecasting the spread of covid-19. ArXiv Preprint ArXiv:2004.04741, 2020.

[17] Xingjie Hao, Shanshan Cheng, Degang Wu, Tangchun Wu, Xihong Lin, and Chaolong Wang. Reconstruction of the full transmission dynamics of covid-19 in wuhan. Nature, 584(7821):420-424, 2020.

[18] Cleo Anastassopoulou, Lucia Russo, Athanasios Tsakris, and Constantinos Siettos. Data-based analysis, modelling and forecasting of the COVID-19 outbreak. PloS one, 15(3):e0230405, 2020.

[19] Liangrong Peng, Wuyue Yang, Dongyan Zhang, Changjing Zhuge, and Liu Hong. Epidemic analysis of covid-19 in china by dynamical modeling. AXiv Preprint ArXiv:2002.06563, 2020.

[20] Benjamin Ivorra, Miriam Ruiz Ferrández, Marıa Vela-Pérez, and AM Ramos. Mathematical modeling of the spread of the coronavirus disease 2019 (covid-19) taking into account the undetected infections. the case of china. Communications In Nonlinear Science And Numerical Simulation, 88:105303, 2020.

[21] Reza Sameni. Mathematical modeling of epidemic diseases; a case study of the covid19 coronavirus. ArXiv Preprint ArXiv:2003.11371, 2020.

[22] Minah Park, Alex R Cook, Jue Tao Lim, Yinxiaohe Sun, and Borame L Dickens. A systematic review of covid-19 epidemiology based on current evidence. Journal Of Clinical Medicine, 9(4):967, 2020.

[23] Flavia Riccardo, Marco Ajelli, Xanthi Andrianou, Antonino Bella, Martina Del Manso, Massimo Fabiani, Stefania Bellino, Stefano Boros, Alberto Mateo Urdiales, Valentina Marziano, et al. Epidemiological characteristics of covid-19 cases in italy and estimates of the reproductive numbers one month into the epidemic. 
MedRxiv, 2020.

[24] IHME Covid. Modeling covid-19 scenarios for the united states. Nature Medicine, 27(1):94, 2021.

[25] Sibel Eker. Validity and usefulness of covid-19 models. Humanities And Social Sciences Communications, 7(1):1-5, 2020.

[26] Joseph Friedman, Patrick Liu, and Emmanuela Gakidou. Predictive performance of international covid-19 mortality forecasting models. MedRxiv, 2020.

[27] Manav R Bhatnagar. Covid-19: Mathematical modeling and predictions. submitted to ArXiv. Online available at: http://web. iitd. ac. in/ manav/COVID. pdf, 2020.

[28] Fotios Petropoulos and Spyros Makridakis. Forecasting the novel coronavirus COVID-19. PloS one, 15(3):e0231236, 2020.

[29] Hamada Badr, Hongru Du, Max Marshall, Ensheng Dong, Marietta Squire, and Lauren Marie Gardner. Social distancing is effective at mitigating COVID-19 transmission in the United States. MedRxiv, 2020.

[30] Giulia Giordano, Franco Blanchini, Raffaele Bruno, Patrizio Colaneri, Alessandro Di Filippo, Angela Di Matteo, and Marta Colaneri. Modelling the covid-19 epidemic and implementation of population-wide interventions in italy. Nature Medicine, pages $1-6,2020$.

[31] Marino Gatto, Enrico Bertuzzo, Lorenzo Mari, Stefano Miccoli, Luca Carraro, Renato Casagrandi, and Andrea Rinaldo. Spread and dynamics of the covid-19 epidemic in italy: Effects of emergency containment measures. Proceedings of the National Academy of Sciences, 117(19):10484-10491, 2020.

[32] Veria Khosrawipour et al. Failure in initial stage containment of global COVID-19 epicenters. Journal Of Medical Virology, 2020. 
[33] Aaron B. Wagner et al. Social distancing merely stabilized COVID-19 in the United States. Stat, 9(1):e302, 2020. e302 sta4.302.

[34] Hien Lau et al. The positive impact of lockdown in wuhan on containing the COVID19 outbreak in China. Journal Of Travel Medicine, 2020.

[35] Kevin Linka, Mathias Peirlinck, Francisco Sahli Costabal, and Ellen Kuhl. Outbreak dynamics of COVID-19 in europe and the effect of travel restrictions. Computer Methods In Biomechanics And Biomedical Engineering, pages 1-8, 2020.

[36] Huaiyu Tian et al. An investigation of transmission control measures during the first 50 days of the COVID-19 epidemic in China. Science, 368(6491):638-642, 2020.

[37] Ming-Tao Li et al. Analysis of COVID-19 transmission in Shanxi Province with discrete time imported cases. Mathematical Biosciences And Engineering, 17(4):3710, 2020.

[38] Centers for Disease Control and Prevention. Interim guidance for antigen testing for sars-cov-2. https://www.cdc.gov/coronavirus/2019-ncov/lab/resources/ antigen-tests-guidelines.html, 2020. [Online; accessed 20-December-2020].

[39] Jessica Watson, Penny F Whiting, and John E Brush. Interpreting a COVID-19 test result. British Medical Association, 369, 2020.

[40] Carl Mayers and Kate Baker. Impact of false-positives and false-negatives in the uk's covid-19 rt-pcr testing programme, 2020.

[41] Nandini Sethuraman, Sundararaj Stanleyraj Jeremiah, and Akihide Ryo. Interpreting diagnostic tests for SARS-CoV-2. Journal Of The American Medical Association, $323(22), 2020$.

[42] Jeffrey P Kahn. Digital Contact Tracing For Pandemic Response: Ethics And Governance Guidance. Johns Hopkins University Press, 2020. 
[43] MS Windows NT kernel description. https://www.pewresearch.org/internet/ 2020/10/30/the-challenges-of-contact-tracing-as-u-s-battles-covid-19/. Accessed: 2010-09-30.

[44] Google LLC. Google COVID-19 community mobility reports. https://www.google.com/covid19/mobility. Last accessed May 18, 2020.

[45] Hamada S Badr, Hongru Du, Maximilian Marshall, Ensheng Dong, Marietta M Squire, and Lauren M Gardner. Association between mobility patterns and covid-19 transmission in the usa: a mathematical modelling study. The Lancet Infectious Diseases, 20(11):1247-1254, 2020.

[46] Caroline O Buckee, Satchit Balsari, Jennifer Chan, Mercè Crosas, Francesca Dominici, Urs Gasser, Yonatan H Grad, Bryan Grenfell, M Elizabeth Halloran, Moritz UG Kraemer, et al. Aggregated mobility data could help fight covid-19. Science (New York, NY), 368(6487):145-146, 2020.

[47] Moritz UG Kraemer, Chia-Hung Yang, Bernardo Gutierrez, Chieh-Hsi Wu, Brennan Klein, David M Pigott, Louis Du Plessis, Nuno R Faria, Ruoran Li, William P Hanage, et al. The effect of human mobility and control measures on the covid-19 epidemic in china. Science, 368(6490):493-497, 2020.

[48] Chong You, Yuhao Deng, Wenjie Hu, Jiarui Sun, Qiushi Lin, Feng Zhou, Cheng Heng Pang, Yuan Zhang, Zhengchao Chen, and Xiao-Hua Zhou. Estimation of the timevarying reproduction number of covid-19 outbreak in china. International Journal Of Hygiene And Environmental Health, page 113555, 2020.

[49] Jane M Heffernan, Robert J Smith, and Lindi M Wahl. Perspectives on the basic reproductive ratio. Journal Of The Royal Society Interface, 2(4):281-293, 2005.

[50] Hyun Mo Yang. The basic reproduction number obtained from jacobian and next generation matrices-a case study of dengue transmission modelling. Biosystems, 
126:52-75, 2014.

[51] Lorenzo Pellis et al. Challenges in control of COVID-19: short doubling time and long delay to effect of interventions. ArXiv Preprint ArXiv:2004.00117, 2020.

[52] Zhanwei Du et al. Risk for transportation of coronavirus disease from Wuhan to other cities in China. Emerging Infectious Diseases, 26(5):1049, 2020.

[53] Tonglin Zhang and Ge Lin. Spatiotemporal analysis for the outbreak of COVID-19 in the world. Available at SSRN 3576816, 2020.

[54] Adam J. Kucharski et al. Early dynamics of transmission and control of COVID-19: a mathematical modelling study. The Lancet Infectious Diseases, 2020.

[55] Paul Hong Lee. Estimating the real-time case fatality rate of COVID-19 using poisson mixtures model. MedRxiv, 2020.

[56] William S Cleveland. Robust locally weighted regression and smoothing scatterplots. Journal Of The American Statistical Association, 74(368):829-836, 1979.

[57] William S Cleveland. Lowess: A program for smoothing scatterplots by robust locally weighted regression. American Statistician, 35(1):54, 1981.

[58] William S Cleveland and Susan J Devlin. Locally weighted regression: an approach to regression analysis by local fitting. Journal of the American statistical association, 83(403):596-610, 1988.

[59] Patrick Royston. Lowess smoothing. Stata Technical Bulletin, 1(3), 1992.

[60] Spencer Woody, Mauricio Garcia Tec, Maytal Dahan, Kelly Gaither, Michael Lachmann, Spencer Fox, Lauren Ancel Meyers, and James G Scott. Projections for firstwave covid-19 deaths across the us using social-distancing measures derived from mobile phones. MedRxiv, 2020.

[61] Francesco Chirico, Gabriella Nucera, and Nicola Magnavita. Estimating case fatality ratio during covid-19 epidemics: pitfalls and alternatives. The Journal Of Infection 
[62] IHME COVID, Christopher JL Murray, et al. Forecasting covid-19 impact on hospital bed-days, ICU-days, ventilator-days and deaths by us state in the next 4 months. MedRxiv, 2020.

[63] Hakan Yilmazkuday. Stay-at-home works to fight against covid-19: international evidence from google mobility data. Journal Of Human Behavior In The Social Environment, pages 1-11, 2020.

[64] Haiyan Wang and Nao Yamamoto. Using a partial differential equation with google mobility data to predict covid-19 in arizona. Mathematical Biosciences and Engineering, 17(5), 2020.

[65] Alexander Lachmann. Correcting under-reported COVID-19 case numbers. MedRxiv, 2020.

[66] Steven G. Krantz and Arni S. R. Srinivasa Rao. Level of under-reporting including under-diagnosis before the first peak of COVID-19 in various countries: Preliminary retrospective results based on wavelets and deterministic modeling. Infection Control \& Hospital Epidemiology, pages 1-8, 2020.

[67] David V Hinkley. Bootstrap methods. Journal of The Royal Statistical Society: Series B (Methodological), 50(3):321-337, 1988.

[68] James Carpenter and John Bithell. Bootstrap confidence intervals: when, which, what? a practical guide for medical statisticians. Statistics In Medicine, 19(9):1141$1164,2000$.

[69] Abdefihak M Zoubir and D Robert Iskandler. Bootstrap methods and applications. IEEE Signal Processing Magazine, 24(4):10-19, 2007.

[70] Anthony Christopher Davison and David Victor Hinkley. Bootstrap Methods And Their Application. Number 1. Cambridge University Press, 1997. 
medRxiv preprint doi: https://doi.org/10.1101/2021.02.01.21250936; this version posted February 3, 2021. The copyright holder for this preprint (which was not certified by peer review) is the author/funder, who has granted medRxiv a license to display the preprint in perpetuity.

It is made available under a CC-BY-NC-ND 4.0 International license.

[71] Michael R Chernick, Wenceslao González-Manteiga, Rosa M Crujeiras, and Erniel B Barrios. Bootstrap Methods, 2011. 